Musées, Patrimoine et Culture scientifiques et techniques

$117 \mid 2008$

mai - juin 2008

\title{
Odyssées en conteneurs
}

\section{Bruno Dosseur}

URL : http://journals.openedition.org/ocim/328

DOI : $10.4000 /$ ocim.328

ISSN : 2108-646X

\section{Éditeur}

OCIM

Édition imprimée

Date de publication : 1 mai 2008

Pagination : 19-26

ISSN : 0994-1908

Référence électronique

Bruno Dosseur, "Odyssées en conteneurs », La Lettre de l'OCIM [En ligne], 117 | 2008, mis en ligne le 01 décembre 2010, consulté le 19 avril 2019. URL : http://journals.openedition.org/ocim/328 ; DOI :

$10.4000 /$ ocim. 328 
farouche d'investir un territoire avec beaucoup d'imagination et de sens pratique. Aujourd'hui, il reste l'élément le plus visible de cette incroyable prouesse technique.

Le clin d'œil à son histoire locale était tentant pour Relais d'sciences, le CCSTI de Basse-Normandie. Ne disposant pas de lieu permanent, Relais d'sciences se posait, depuis sa création en 1998, la question de sa capacité à proposer une offre culturelle sur l'ensemble du territoire régional (1). Comment investir un espace de plus de $17000 \mathrm{~km}^{2}$, s'identifier auprès d'un million et demi d'habitants (sans compter les très nombreux touristes), ancrer une démarche pérenne de diffusion d'une offre de culture scientifique et technique, parfois à plus de cent kilomètres du siège du CCSTI ?

De 1998 à 2003, Relais d'sciences adopte une première stratégie de développement par la mise en place d'antennes dans une demi-douzaine de villes en BasseNormandie. Chaque antenne comprend un médiateur scientifique disposant d'un local permanent. Malgré d'incontestables réussites, ce schéma de diffusion se heurte vite à la difficulté de définir une offre claire et des objectifs partagés avec les acteurs locaux. L'absence d'une identité régionale affirmée est compliquée par le management à distance d'une équipe jeune et sans réels moyens d'action.

Après une phase de regroupement et de consolidation du siège (2004-2006) le CCSTI élabore une nouvelle stratégie fondée sur les publics à atteindre. En quelques années, l'équipe de Relais d'sciences apprend à investir les locaux de ses partenaires (CCI d'Alençon, université de Caen, Conseil général de l'Orne, planétarium Ludiver...) pour y monter des opérations de plusieurs mois. Jonglant avec des espaces sans cesse différents, pas toujours adaptés ou disponibles, elle identifie l'intérêt d'une programmation ambitieuse dont pourraient se saisir ses partenaires locaux.

Le projet de Relais d'sciences est de susciter la curiosité des publics, de les amener à s'intéresser au monde qui les entoure dans toutes ses dimensions. Sa démarche s'ancre dans l'action culturelle afin d'accéder aux publics et mettre chacun en état de recherche et d'interrogations. De cette réflexion est né un cycle de trois années baptisé Odyssées.

\section{Odyssées, l'esprit voyage}

Odyssées est le fruit d'un long travail de réflexion mené au sein de l'équipe du CCSTI. L'objectif était
Relais d'sciences, le CCSTI de Basse-Normondie

Le CCSTI de Basse-Normandie est né en 1998 à l'initiative de l'État et de la Région BasseNormandie. Il est constitué autour de cinq grands partenaires scientifiques : la délégation Normandie du CNRS, la plateforme d'Imagerie biomédicale CYCERON, l'École nationale supérieure d'Ingénieurs de Caen et Centre de Recherche (ENSICAEN), le Grand Accélérateur national d'Ions Lourds (GANIL) et l'Université de Caen Basse-Normandie. Il travaille également en lien étroit avec les acteurs culturels et éducatifs.

Fort d'une équipe de dix permanents Relais d'sciences (www.relaisdsciences.org) déploie ses opérations dans le Calvados, la Manche et l'Orne. En plus de sa programmation Odyssées, il propose un cycle de bars des sciences à Caen, organise la Fête de la Science et l'Exposciences et accompagne les acteurs de la culture scientifique en région dans leurs projets à destination du public. Relais d'sciences a été déclaré d'intérêt général en 2005 et labellisé "Science et Culture, Innovation » en 2008.

de définir une offre culturelle, programmée à l'année, lisible par tous, répondant aux attentes de publics différents et accessible en plusieurs points du territoire régional (www.odyssee-blanche.org).

Le Petit Robert définit une odyssée comme "le récit d'un voyage rempli d'aventures ». Le projet se lit donc avec une double dimension : la mobilité indispensable à l'itinérance et le dépassement des limites connues, avec tous les risques et les bénéfices que cela comporte. Ce voyage va conduire les publics vers trois destinations : les pôles (Odyssée blanche, 2007/2008), les déserts (Odyssée jaune, 2008/2009) et les forêts tropicales (Odyssée verte, 2009/2010). Trois espaces encore mal connus, propres à l'imagination et à l'esthétisme, trois laboratoires à ciel ouvert où se croisent scientifiques et peuples autochtones, trois symboles d'une planète en pleine évolution.

Les Odyssées permettent d'aborder toutes les sciences de la nature, les sciences humaines et sociales. Elles se font l'écho de la recherche fondamentale comme des applications technologiques les plus 
avancées. Elles évoquent l'aventure humaine et les défis scientifiques. Par la beauté des espaces présentés et leur charge émotive, elles favorisent le croisement avec le regard des artistes. L'ambition est multiple :

- emmener le visiteur dans un voyage, prétexte à une découverte et, pourquoi pas, à une expérience inédite ; - interpeller le public en bousculant les limites de son univers connu ;

- susciter l'envie et le plaisir d'explorer, d'interroger, et, finalement, de venir vivre l'expérience suivante ; - finalement créer un public, avec toute sa diversité culturelle, sociale ou géographique.

Travailler dans la durée à plus de 100 kilomètres de sa base nécessite de s'appuyer sur des partenaires locaux mais également de proposer un rendez-vous clair et récurrent au public. Tout comme un festival n'existe que par sa capacité à revenir et à innover chaque année, Odyssées s'implanterait dans le territoire par sa pérennité et son originalité. Pour donner corps à cette ambition, Relais d'sciences devait créer un lieu de visibilité. Un lieu d'attraction et de diffusion. Un lieu proche des publics mais également source d'inspiration pour les acteurs culturels, éducatifs et institutionnels sensibles au thème proposé. Un lieu immédiatement identifiable et surprenant.

L'Odyssée blanche, première du cycle Odyssées, imposait pratiquement la réponse. Le premier défi de l'aventure scientifique aux pôles est une histoire d'hommes, amenés à survivre dans un milieu hostile. En 1956, la France construit la base de recherche Dumont d'Urville en Antarctique, sur l'île des Pétrels.

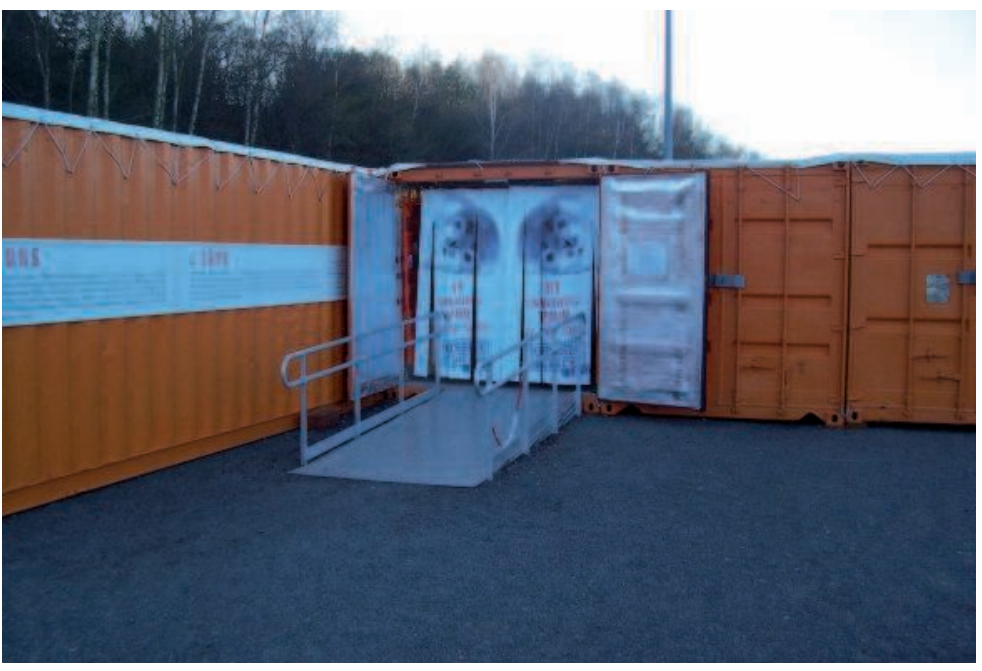

L'entrée de l'exposition (c) Relais d'sciences
Cette base est constituée en grande partie de conteneurs, acheminés par voie maritime et immédiatement identifiables par leur couleur orange. Les bâtiments ainsi réalisés servent d'ateliers, de lieux de stockage ou de laboratoires.

En utilisant les mêmes conteneurs pour évoquer la base Dumont d'Urville, Relais d'sciences pouvait concilier contenant et contenu et donner à l'exposition Odyssée blanche tout son sens.

\section{Un défi technique pour un lieu phare}

Construire de toutes pièces un tel lieu était d'abord un pari technique en raison des nombreuses inconnues. Des architectes et des concepteurs d'expositions d'art contemporain utilisent des conteneurs dans leurs réalisations depuis plusieurs années. Mais il n'est pas aisé pour un CCSTI d'accéder aux enseignements techniques et fonctionnels qu'ils tirent de ces expériences. Il a donc fallu improviser face aux nombreuses questions restées en suspens. Comment assurer une parfaite jonction et l'étanchéité entre les conteneurs si le sol n'est pas tout à fait plan ? Pourra-t-on s'entendre avec trente élèves au milieu de ces parois métalliques ? Et s'il fait trop chaud ou trop froid ? Comment garantir le fonctionnement des appareils en cas de gel ? Le responsable du développement culturel à Relais d'sciences a imaginé le dispositif et en a tracé les plans à l'aide du logiciel 3D freeware Google SketchUp. Cet outil interactif a facilité la conception et a permis des présentations vidéoprojetées de grande qualité lors de la rencontre avec les partenaires de l'opération.

Les conteneurs marins sont comme des pièces de legos. En un peu plus gros. Leur intérêt réside dans leur solidité et leur étonnante mobilité, malgré leur poids. Leur conception permet de les accoler pour créer des salles, de les placer pour former un patio ou un couloir, voire de les superposer afin d'obtenir des volumes en hauteur. Leur longueur peut être de 10 pieds (environ 3 mètres), 20 pieds (6 mètres) ou 40 pieds (12 mètres) pour une largeur unique de 2,44 mètres. La hauteur intérieure est en moyenne de 2,34 mètres. Le poids d'un conteneur vide de 40 pieds est environ de 3,5 tonnes.

Afin d'obtenir un espace d'accueil billetterie et une surface d'exposition de $200 \mathrm{~m}^{2}$, il fallait assembler neuf conteneurs : six conteneurs de 40 pieds pour constituer trois salles de $60 \mathrm{~m}^{2}$, un conteneur de 40 pieds pour réaliser un couloir reliant les salles et deux conteneurs de 20 pieds pour constituer l'accueil. 


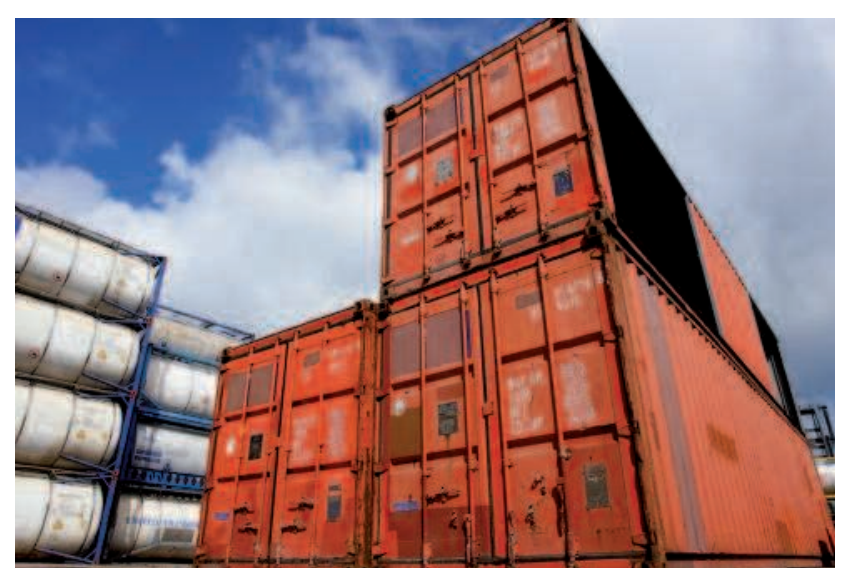

Des conteneurs marins comme à la base Dumont d'Urville (C) Relais d'sciences

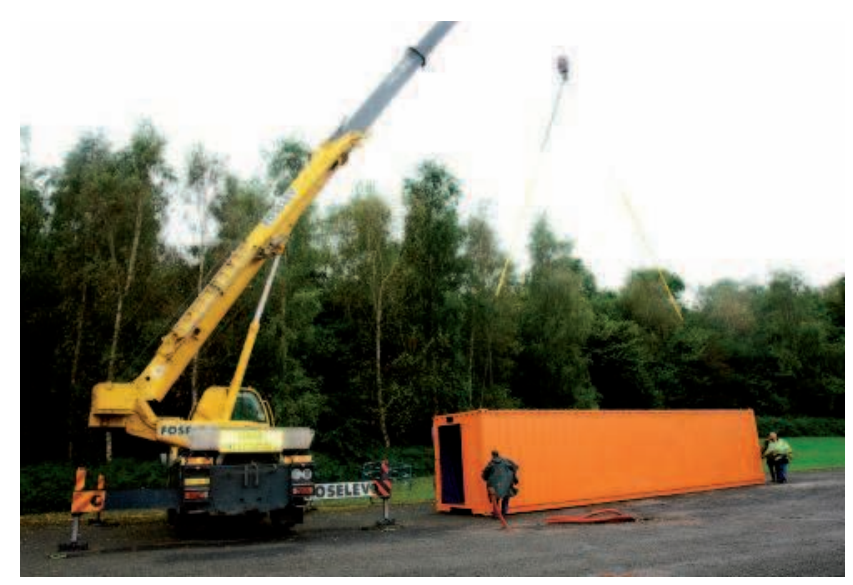

Installation (C) Relais d'sciences

\section{Deux mois de chantier au chevet des conteneurs}

Pour garantir la faisabilité du projet, Relais d'sciences s'est entouré d'entreprises compétentes choisies en région. Le gros œuvre a été confié à une société basée au Havre, spécialisée dans la configuration des conteneurs à des fins industrielles et de transport. Elle a sélectionné pour Relais d'sciences neuf conteneurs d'occasion et a assuré leur transformation et leur transport sur chacun des sites de l'exposition.

Le plus gros chantier consistait à transformer les conteneurs : ouverture des portes et des parois latérales pour constituer les assemblages en salle, installation de murs et de planchers, isolation thermique, câblage électrique et pose de chauffages électriques, peintures intérieures et extérieures.

Une fois reconfigurés, les conteneurs ont été acheminés à Caen en un long convoi de poids lourds. Le

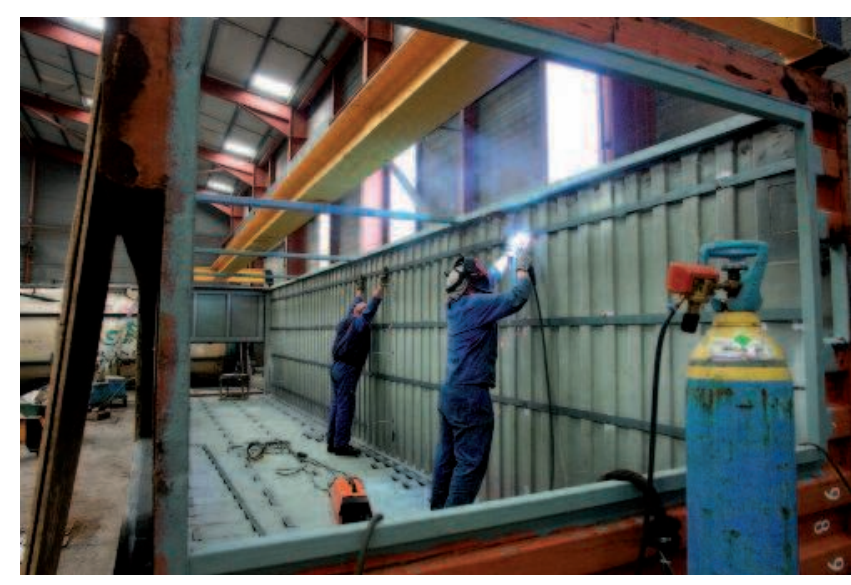

Préparation (C) Relais d'sciences



Aménagement (C) Relais d'sciences

grutage et la mise en place au cour du parc floral municipal dit de la «Colline aux Oiseaux » a pris une journée. Le tout rythmé par une pluie diluvienne dont nous avons le secret en Normandie. Sous l'œil étonné des médias locaux, le dispositif a rapidement pris forme et constitué un bâtiment original au cœur de la verdure.

L'ensemble doit s'appuyer sur un terrain stabilisé du fait de ses 30 tonnes et être installé de façon parfaitement plane pour assurer une parfaite jonction entre les conteneurs. Des cales sont donc nécessaires pour corriger les défauts du terrain. Une bâche vient couvrir l'ensemble de la toiture pour renforcer l'étanchéité et réduire l'impact sonore de la pluie. Une rampe mobile garantit l'accès aux personnes à mobilité réduite.

Une seconde phase d'aménagement intérieur a permis la mise en place des liaisons électriques, d'un système d'alarme, des dispositifs incendie (blocs 
issue de secours et extincteurs), de l'ensemble des éléments audiovisuels, d'une régie vidéo et son, de l'informatisation de l'accueil avec logiciel de billetterie, imprimante à billet, coffre-fort, connexion Internet $\mathrm{G} 3+$, terminal de paiement bancaire... La régie vidéo compte dix neuf sources de diffusion alimentant quatorze écrans muraux, un écran cinéma de $12 \mathrm{~m}^{2}$, quatre vidéoprojections au sol et au plafond. Plusieurs sources sonores directes ou d'ambiance ponctuent le parcours de visite. Bien entendu, tous ces éléments doivent être parfaitement fixés ou, au contraire, facilement démontables pour permettre le déplacement du dispositif d'une ville à l'autre en très peu de temps.

À l'issue de ce chantier de deux mois, nous nous trouvons en face d'un bâtiment inédit, parfaitement inviolable et résistant aux pires intempéries. Un raccordement électrique suffit à le mettre en service. Cette autonomie garantit sa parfaite intégration en tout lieu extérieur plat et accessible par poids lourds.

\section{Mise en scène de la première Odyssée}

Relais d'sciences dispose désormais d'un lieu pour accueillir le public. La force du dispositif réside bien sûr dans sa mobilité et son autonomie mais également dans la totale liberté quant à son aménagement intérieur. Destiné à accueillir successivement les trois Odyssées (blanche, jaune et verte), le lieu peut accueillir 80 personnes en même temps et se trouve particulièrement adapté pour les groupes scolaires.

Sur cette base, les médiateurs scientifiques de Relais d'sciences ont travaillé pendant une année à

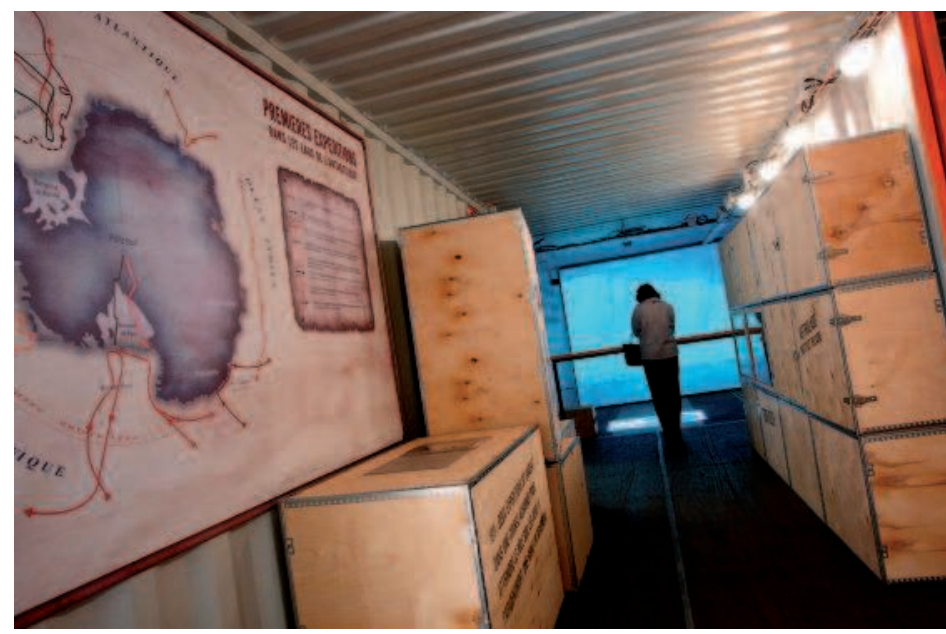

Les premières expéditions et la découverte des paysages () Relais d'science la conception de l'exposition Odyssée blanche : une découverte de la base scientifique polaire Dumont d'Urville et des travaux qui y sont menés. Les médiateurs scientifiques voulaient un parti pris fort traduisant leur volonté de faire participer le visiteur à sa propre visite. La scénographie est donc volontairement originale pour susciter une démarche nouvelle. Les panneaux sont remplacés par des objets et des sources sonores portant l'information, les expériences sont nichées au cœur de réfrigérateurs thématisés. Des animateurs scientifiques sont présents en permanence pour accompagner les publics. Plus d'une vingtaine d'équipes scientifiques françaises et d'organismes publics ont été sollicités pour la préparation d'Odyssée blanche dont l'Institut polaire français Paul-Émile Victor (IPEV), pilote national de l'Année Polaire Internationale.

Le travail de médiation a conduit à la mise en place de quatre espaces au sein du dispositif. Le choix des matériaux et les passages de câbles électriques apparents accentuent l'effet d'immersion dans des locaux à vocation technique tout en conservant la qualité esthétique de l'ensemble:

- la première salle est dédiée au voyage à bord du navire Astrolabe qui amène les équipes françaises en Terre Adélie. Les premières explorations sont évoquées, notamment à travers lincroyable épopée de Shakelton. Volontairement, la salle n’a pas été isolée afin de garantir une température assez basse dans un décor de cale de bateau. L'effet est presque trop bien réussi en plein hiver avec une température proche de zéro !

- un couloir dessert ensuite les deux autres espaces de visite. Il évoque les paysages de la base scientifique, notamment grâce à des visuels de grande taille spécifiquement réalisés en Antarctique.

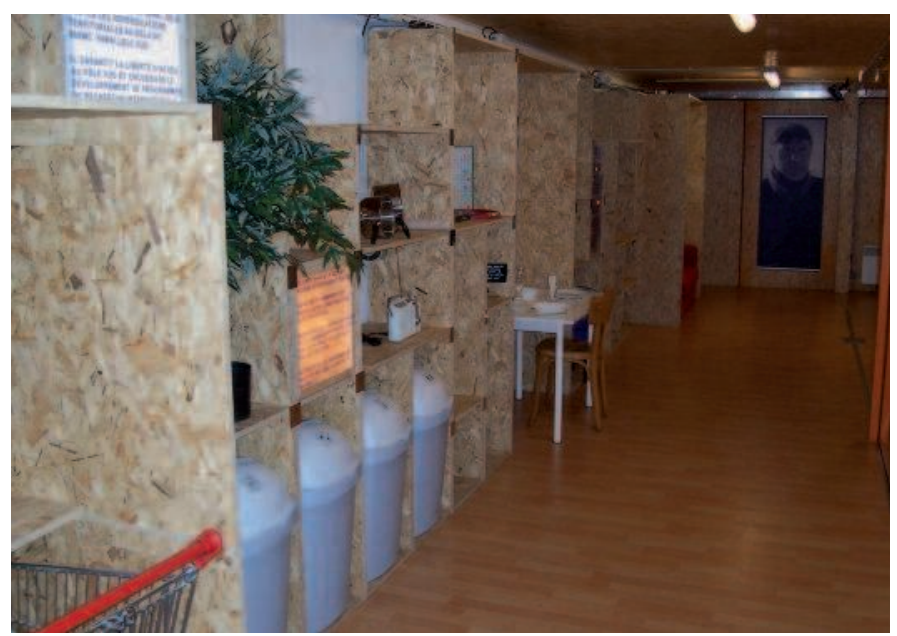

La salle consacrée à la vie quotidienne des occupants de la base Dumont d'Urville (c) Relais d'sciences 
- une seconde salle plonge le visiteur dans le quotidien des habitants de la base. Celui-ci est invité à s'emparer des objets, à les manipuler, à rechercher les sources sonores afin d'accéder aux informations. - la dernière salle présente, à travers de surprenants réfrigérateurs, les principaux thèmes des recherches menées à Dumont d'Urville et sur la toute nouvelle base franco-italienne de Concordia : étude des manchots, glaciologie, astronomie, micro-météorites, magnétisme, couche d'ozone.

À l'extérieur du bâtiment, les visiteurs sont invités à découvrir "Stations », une œuvre de Catherine Rannou, basée sur des vidéogrammes réalisés en résidence à Dumont d'Urville.

\section{Une programmation culturelle}

Dès sa conception, l'Odyssée a eu pour vocation la mise en place d'une programmation culturelle sur les pôles à l'attention de tous les publics. Elle ne saurait donc se résumer uniquement au dispositif d'exposition même si, incontestablement, celui-ci constitue le cœur de l'opération... la partie immergée de l'iceberg.

Les trois départements bas-normands reçoivent donc successivement la même proposition : l'exposition en conteneurs, un cycle de rencontres avec les meilleurs spécialistes des questions polaires et des outils d'animation. Ce dernier volet permet aux acteurs d'un territoire local de se saisir de la thématique des pôles pour créer leur propre programmation. Il comprend quatre propositions pour des publics différents et complémentaires de ceux de l'exposition :

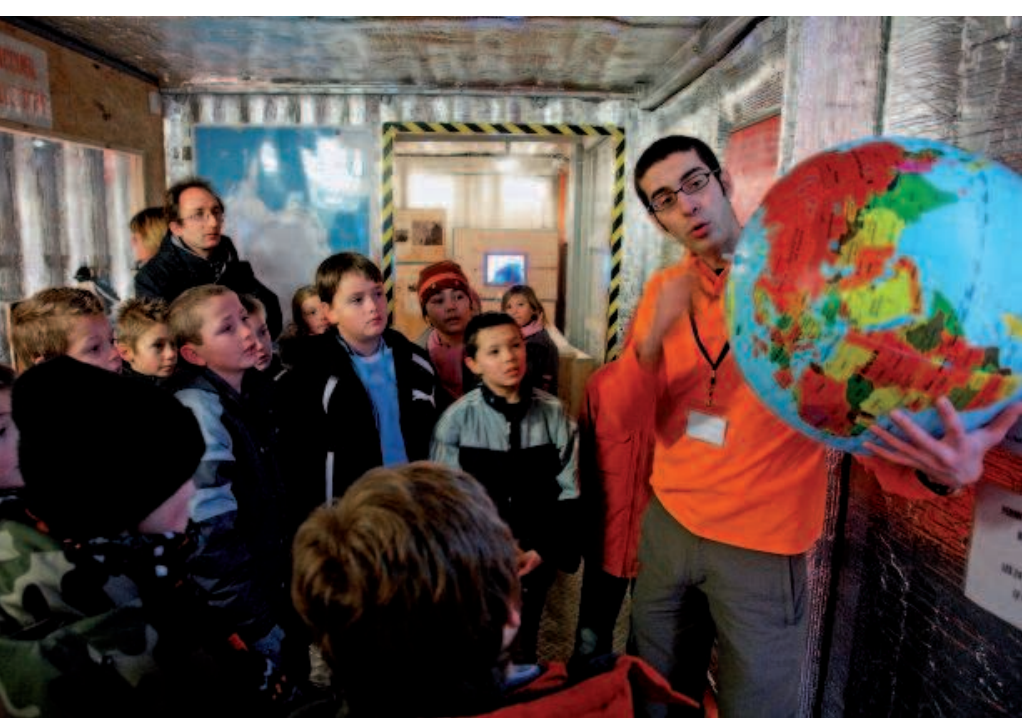

Des animations pour les scolaires () Relais d'sciences
- «Odyssée au long cours » : un travail de fond avec des classes de SEGPA (2), inspiré du Passeport Découverte de la Fondation 93, donnant lieu à des projets présentés dans le cadre de l'Exposciences régionale ;

- « Du nord au Sud » : un outil itinérant à l'attention des familles proposant de découvrir, avec des animateurs de Relais d'sciences, les différences entre les pôles ;

- «L'Odyssée du livre »: une collaboration avec les bibliothèques-médiathèques et la DRAC de Basse-Normandie pour favoriser l'achat et la mise à disposition du public d'ouvrages de science et technique en lien avec le thème de l'Odyssée ;

- "Pôles en direct », un site Internet entièrement dédié à l'Odyssée blanche proposant notamment des carnets de route d'hivernants et des informations détaillées sur les pôles.

Afin de renforcer l'impact de l'Odyssée sur chaque territoire, Relais d'sciences s'est efforcé de concentrer les opérations sur un même département pendant toute la durée de présentation de l'exposition dans l'agglomération la plus importante (Caen, Alençon puis Cherbourg). Les publics pouvaient donc approfondir leur découverte des pôles en rencontrant Jean Malaurie, Jean Duprat ou Christine David, retrouver des ouvrages en bibliothèque, ou retrouver près de chez eux une animation familiale sur les pôles.

De très nombreuses collaborations ont été nécessaires pour mettre en œuvre un programme aussi dense. En plus des scientifiques, une dizaine d'artistes se sont mobilisés ainsi que des centres culturels comme l'institut Mémoires de l'Édition contemporaine (IMEC), le centre régional des Lettres dans le cadre du festival des Boréales, le fond régional d'Art Contemporain ou le café des Images, cinéma d'art et d'essai.

Certains partenaires ont pris l'initiative de monter leurs propres opérations sur le thème des pôles afin de s'inscrire dans la dynamique de l'Odyssée. Ainsi la Comédie de Caen, centre dramatique national de Normandie, a programmé pendant une semaine Le pendule polaire, une scénographie vidéo et sonore d'Anne Durez et Jean-Luc Therminarias. Le musée de la Poste et des Techniques de Communication de Caen s'est emparé du sujet pour réaliser une exposition sur la philatélie dans les Terres Australes et Antarctiques Françaises. Ludiver, le planétarium de La Hague, programme des découvertes du ciel polaire en lien avec l'arrivée de l'exposition à Cherbourg. 
Grâce à l'ambition de sa proposition culturelle, la démarche proposée dans le cadre d'Odyssées a reçu de nombreux soutiens financiers. La Région BasseNormandie accompagne l'opération dans le cadre d'un contrat d'objectifs à trois ans afin de garantir son développement sur l'ensemble du territoire. Des entreprises et organismes interviennent au titre du mécénat culturel, à l'image de l'ANDRA (3), pour favoriser la diffusion d'opérations de culture scientifique. Enfin, l'Agence nationale de la Recherche, La Cité de la Mer de Cherbourg et les villes de Caen et Alençon soutiennent spécifiquement l'Odyssée blanche dans le cadre de l'Année Polaire Internationale.

\section{Moins d'une semaine pour changer de ville}

Après quatre mois de présentation à Caen, l'Odyssée blanche a quitté la capitale régionale en février 2008 pour rejoindre Alençon. Elle est proposée par La Cité de la Mer de Cherbourg jusqu'à la fin du mois d'août $20088^{(4)}$.

L'intérêt et la fiabilité du dispositif ont donc pu être testés entre Caen et Alençon. Désormais, l'exposition peut fermer ses portes au public un dimanche soir dans une ville et ouvrir le week-end suivant dans une autre ville, voire une autre région. Une semaine suffit pour assurer le déplacement de tout le bâtiment avec son dispositif scénographique, ses 19 éléments vidéo, ses éclairages, sa régie et sa billetterie embarquées. L'installation d'une exposition se heurte toujours aux impondérables du lieu d'accueil. Il faut adapter la scénographie aux salles, repenser l'éclairage, installer les équipements audiovisuels, repenser les déplacements des visiteurs...

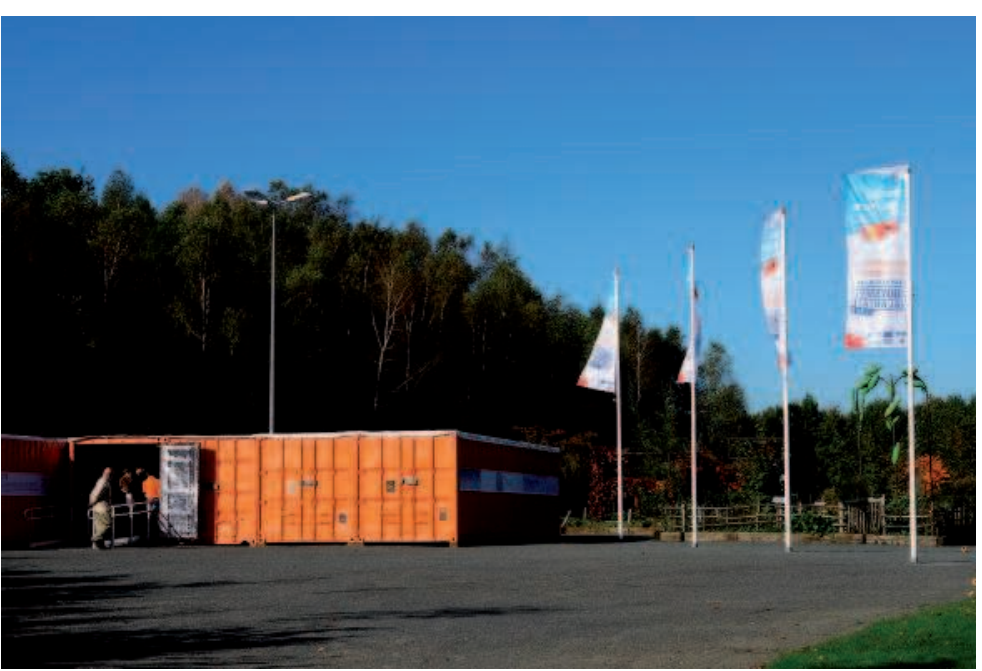

Une installation facile et adaptée à tous les lieux (C) Relais d'sciences
Le dispositif de conteneurs lève la contrainte de temps imposée dans la plupart des lieux culturels du fait de l'enchaînement des expositions. L'autonomie du lieu permet de choisir en toute liberté la durée de présentation au public. La mobilité est facilitée par de faibles coûts marginaux d'exploitation. Le déplacement du bâtiment entre deux préfectures d'une même région représente en effet moins de $2 \%$ du coût total de l'opération. Le dernier avantage est la solidité du contenant. Inviolable du fait même de sa conception, insensible au mauvais temps, il peut être installé sur tout terrain stabilisé disposant d'un accès électrique. Idéal pour animer un parc, une place de centre ville, un port, il ne nécessite aucun gardiennage.

L'équipe d'animation suit l'exposition pour la faire vivre et déployer les formats d'animation destinés aux autres communes du département. La communication est organisée à l'échelle départementale et s'appuie autant sur le contenu que sur le contenant. Enfin, la mobilisation des élèves est suivie à l'échelle académique grâce à une centrale de réservation sur Internet.

\section{Vers d'autres odyssées}

L'équipe de Relais d'sciences travaille désormais sur les deux autres Odyssées, jaune et verte en attendant un nouveau cycle de trois années. La scénographie sera intégralement repensée pour continuer de surprendre tout en conservant les grands principes scénographiques et techniques.

Les possibilités sont nombreuses et avant tout limitées par la créativité et d'incontournables considérations budgétaires. La forme même du dispositif peut être modifiée par une nouvelle organisation des conteneurs au sol, l'ajout ou la superposition de modules, la création d'espaces extérieurs entourés de conteneurs, l'habillage extérieur de l'ensemble ou le choix de son implantation. À l'intérieur, les équipements sont faciles à déplacer et à raccorder à la régie. Les murs et les sols peuvent être repeints ou habillés à l'envie, des sièges peuvent être installés dans un esprit de salle de projection, des éclairages sélectifs et des sources sonores installés pour créer des ambiances particulières. Au lieu d'accueil du public s'ajoute une dimension de lieu de création et d'expérimentation propre à toute démarche culturelle.

\section{Un lieu pour un territoire}

Les professionnels de la diffusion de la culture scientifique en charge de l'animation d'un territoire s’interrogent souvent sur la nécessité de disposer 
d'un lieu permanent et visible pour mener leurs opérations. La construction d'un bâtiment apparaîtra souvent comme la solution privilégiée. Un système d'animation itinérant le complétera parfois pour éviter la centralisation de l'activité sur une seule ville. Certaines structures choisissent à l'inverse le tout « hors murs » et renoncent à la sédentarisation de leur activité pour la plus complète mobilité.

Le dispositif mis en place par Relais d'sciences permet de sortir de ce dilemme du tout fixe ou tout mobile puisqu'il est les deux à la fois. Le coût marginal de son déplacement le rend accessible à toute collectivité de taille moyenne. Il donne un point d'ancrage à une programmation culturelle facilement complétée par des initiatives locales.

Sur le plan symbolique, il porte une égalité de traitement des publics en offrant à chaque collectivité la possibilité de recevoir la même qualité de proposition culturelle, sans nécessiter la présence préalable d'un équipement d'accueil.

Par sa forme originale, il favorise la curiosité. Et, une fois passée l'interrogation légitime des institutions locales, il offre aux publics une proposition singulière et sans cesse renouvelable. Il peut dès lors servir de tête de pont à la diffusion de la culture scientifique en région.

\section{Notes}

(1) Nous n'évoquerons pas ici le second rôle du CCSTI : l'animation du réseau régional à travers la coordination d'opérations régionales (Fête de la Science ou Exposciences) ou l'appui au développement d'actions de culture scientifique chez ses partenaires.

(2) Section d'Enseignement Général et Professionnel Adapté

(3) Agence nationale pour la gestion des déchets radioactifs

(4) À partir de septembre 2008 et jusqu’à la fin de l'année 2008, l'Odyssée blanche sera disponible pour une diffusion en France et dans les pays limitrophes francophones. 